\title{
Effects of cellular origin on differentiation of human induced pluripotent stem cell-derived endothelial cells
}

\author{
Shijun Hu, ${ }^{1,2,3,4}$ Ming-Tao Zhao, ${ }^{1,2,3}$ Fereshteh Jahanbani, ${ }^{5}$ Ning-Yi Shao, ${ }^{1,2,3}$ Won Hee Lee, ${ }^{1,2,3}$ \\ Haodong Chen, ${ }^{1,2,3}$ Michael P. Snyder, ${ }^{5}$ and Joseph C. Wu ${ }^{1,2,3}$ \\ ${ }^{1}$ Stanford Cardiovascular Institute, ${ }^{2}$ Department of Medicine, Division of Cardiology, and ${ }^{3}$ Institute for Stem Cell Biology \\ and Regenerative Medicine, Stanford University School of Medicine, Stanford, California, USA. ${ }^{4}$ Institute for Cardiovascular \\ Science, Soochow University \& Department of Cardiovascular Surgery of the First Affiliated Hospital, Suzhou, Jiangsu, \\ China. ${ }^{5}$ Department of Genetics, Stanford University School of Medicine, Stanford, California, USA.
}

Human induced pluripotent stem cells (iPSCs) can be derived from various types of somatic cells by transient overexpression of 4 Yamanaka factors (OCT4, SOX2, C-MYC, and KLF4). Patient-specific iPSC derivatives (e.g., neuronal, cardiac, hepatic, muscular, and endothelial cells [ECs]) hold great promise in drug discovery and regenerative medicine. In this study, we aimed to evaluate whether the cellular origin can affect the differentiation, in vivo behavior, and single-cell gene expression signatures of human iPSC-derived ECs. We derived human iPSCs from 3 types of somatic cells of the same individuals: fibroblasts (FB-iPSCs), ECs (EC-iPSCs), and cardiac progenitor cells (CPCiPSCs). We then differentiated them into ECs by sequential administration of Activin, BMP4, bFGF, and VECF. EC-iPSCs at early passage $(10<P<20)$ showed higher EC differentiation propensity and gene expression of EC-specific markers (PECAM1 and NOS3) than FB-iPSCs and CPC-iPSCs. In vivo transplanted EC-iPSC-ECs were recovered with a higher percentage of $\mathrm{CD} 31^{+}$population and expressed higher EC-specific gene expression markers (PECAM1, KDR, and ICAM) as revealed by microfluidic single-cell quantitative PCR (qPCR). In vitro EC-iPSC-ECs maintained a higher CD31 population than FB-iPSC-ECs and CPC-iPSC-ECs with long-term culturing and passaging. These results indicate that cellular origin may influence lineage differentiation propensity of human iPSCs; hence, the somatic memory carried by early passage iPSCs should be carefully considered before clinical translation.

Authorship note: S. Hu and M.T. Zhao contributed equally to this work.

Conflict of interest: The authors have declared that no conflict of interest exists.

Submitted: November 16, 2015 Accepted: May 4, 2016

Published: June 2, 2016

Reference information: JCI Insight. 2016;1(8):e85558. doi:10.1172/jci.insight.85558.

\section{Introduction}

Coronary artery disease (CAD) is the most common cause of mortality worldwide. In the United States, about one-fifth of the population over 65 years old has CAD, which contributes to about 1 of every 7 deaths (1). Endothelial dysfunction is considered a key early event in the development of atherosclerosis, which is the primary cause of CAD and myocardial infarction (2). Endothelial cells (ECs) line the interior surface of blood vessels and form a semiselective barrier between the vascular lumen and adjacent tissue. Some ECs have direct contact with blood and serve as immediate sensors and effectors of drug response in the circulation system. Therefore, ECs have been regarded as a useful in vitro model for drug testing in cardiovascular disease. Human umbilical vein/artery ECs are extensively used for studying the function and pathology of ECs in normal and stressed conditions (3). However, they are not patient specific and cannot represent the individual discrepancies observed among patients when used for disease modeling and drug screening. By contrast, genetically matched stem cell-derived ECs can be patient specific and disease specific and are ideal cell sources for investigating the pathological development of CAD and regenerating the blood vessels for purposes of personalized medicine $(4,5)$. For these reasons, patient-specific stem cell-derived ECs and cardiomyocytes would be good candidates for preclinical drug discovery and regenerative therapy for cardiovascular diseases (6).

Human pluripotent stem cells (PSCs) are capable of unlimited self-renewal and multiple-lineage differentiation. Somatic cells can be reprogrammed to the pluripotent state by a number of methods such as cell fusion $(7,8)$, somatic cell nuclear transfer (SCNT) by enucleated oocytes $(9,10)$, and ectopic overex- 
pression of 4 transcription factors (OCT4/SOX2/C-MYC/KLF4) $(11,12)$. The transcription factor-based method has been widely utilized because it circumvents ethical issues stemming from using human oocytes. The resulting cells are known as induced PSCs (iPSCs), which can be derived in a patient- and disease-specific manner and hold great promise for regenerative medicine. Despite subtle differences in epigenetic modifications and gene expression signatures, human iPSCs are generally similar to embryonic stem cells (ESCs) with regard to capacity for unlimited self-renewal and pluripotency $(13,14)$.

Different types of somatic cells carry the epigenetic memory to maintain their tissue-specific cell identities. Because human iPSCs are originally derived from somatic cells, tissue-specific epigenetic memory has been observed in early passage iPSCs (15-18). Recent studies have shown that human iPSCs are equivalent to genetically matched ESCs, and genetic background primarily contributes to the transcriptional variations seen among human ESCs and iPSCs $(19,20)$. However, most of these studies did not test the influence of cellular origin on human iPSC-derived terminally differentiated cells.

To assess whether the donor cell sources can influence the differentiation, in vivo behavior, and gene expression profiles of human iPSC derivatives, we derived iPSCs from 3 types of somatic cells of the same individuals: fibroblasts (FBs), ECs, and cardiac progenitor cells (CPCs). We then compared molecular characteristics and cellular behaviors of ECs derived from these iPSCs (FB-iPSCs, EC-iPSCs, and CPC-iPSCs) both in vitro and in vivo.

\section{Results}

Donor cell sources and iPSC generation. To avoid the genetic effects on the transcriptional program and functional behaviors of iPSCs, we generated isogenic iPSC lines derived from multiple types of donor cells of the same individuals. The experimental design is outlined in Figure 1A. Two aborted human fetuses were collected with the parents' consent (one fetus was at 20 weeks and the other was at 12 weeks of gestation). Genetic screening showed that the parents did not carry any known inherited cardiovascular diseases (data not shown). We isolated ECs from the aorta, FBs from the skin, and CPCs from the heart. We further characterized these 3 types of donor cells to confirm their identity. The fetal ECs showed typical cobblestone-like morphology and highly expressed EC-specific markers CD31 and CD144 (Figure 1, B and C). The fetal ECs have high percentage of $\mathrm{CD} 31^{+}$and $\mathrm{CD} 144^{+}$populations in the culture (Figure 1D). The fetal FBs expressed vimentin, which is a type III intermediate filament specifically enriched in mesenchymal cells (Figure 1, E and F). Human CPCs were isolated and enriched by using magnetic beads conjugated with Sca-1 (stem cell antigen-1) antibody. The Sca- $1^{+}$CPCs expressed CD105 (endoglin) (ref. 21 and Figure $1 G$ ) but were negative for CD133 (hematopoietic stem cell marker), CD34 (endothelial progenitor cell marker), or CD45 (leukocyte marker) (refs. 22-24 and Supplemental Figure 1A; supplemental material available online with this article; doi:10.1172/jci.insight.85558DS1). Human Sca- $1^{+}$CPCs were multipotent and could differentiate into all 3 cardiac lineages under differentiation circumstance, as evidenced by the presence of $\alpha$-actinin ${ }^{+}$cells (cardiomyocytes), $\alpha$-SMA ${ }^{+}$cells (smooth muscle cells), and CD31 ${ }^{+}$cells (ECs) in the differentiation progeny (Supplemental Figure 1, B-D).

Subsequently, we generated isogenic iPSCs from different cell types (CPC, FB, and EC) by lentivirus infection with Yamanaka factors OCT4, SOX2, KLF4, and C-MYC. The resulting iPSCs were called CPC-iPSCs, FB-iPSCs, and EC-iPSCs, respectively. The primary cells were around passage 3-5 when initiating iPSC reprogramming (Supplemental Figure 2A). We generated 2 iPSC lines for each donor cell type: 2 FB-iPSC lines, 2 CPC-iPSC lines, and 2 EC-iPSC lines. The reprogramming efficiencies were slightly different among donor cell types, with CPCs showing the highest efficiency $(0.31 \%)$, FBs showing the lowest efficiency (0.16\%), and ECs showing an intermediate efficiency (0.19\%) (Supplemental Figure 2B). The iPSCs exhibited typical ESC-like colony morphology and were positive for human ESC markers such as TRA-1-60, SSEA4, and alkaline phosphatase (AP) (Supplemental Figure 2C).

The iPSC colonies were then transferred to feeder-free Matrigel-coated plates in mTeSR medium. The established iPSC lines (passage 15) were positive for ESC-specific markers TRA-1-60 and SSEA4, as well as pluripotency factors NANOG and OCT4 (Figure 2A). Karyotype analysis demonstrated stable chromosomal integrity without any transformation in all tested iPSC lines (Figure 2B). Bisulfite sequencing analysis revealed that the promoter region of NANOG was substantially demethylated in all iPSC and ESC lines but was highly methylated in donor cells (Figure 2C). Quantitative PCR (qPCR) demonstrated activation of endogenous pluripotent transcription factors and silencing of lentiviral transgenes (Figure 2D and Supplemental Figure 1E). In addition, these iPSC lines spontaneously formed embryoid bodies (EBs) in the 
A

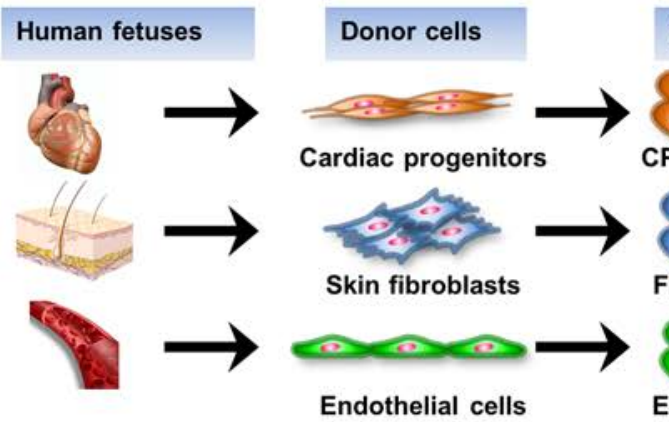

iPSCs
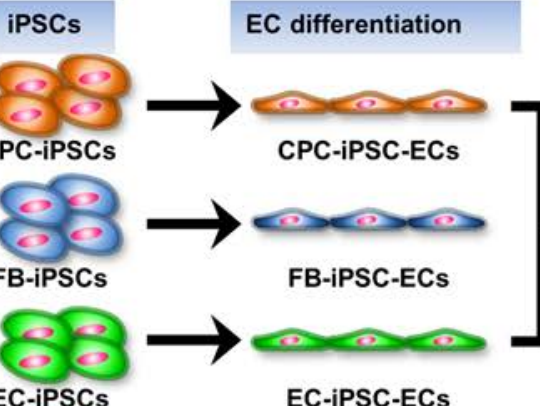

PC-iPSCs

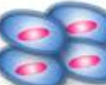

FB-iPSCs

Endothelial cells

EC-iPSCs

EC-iPSC-ECs

Effects of cellular origin on iPSC differentiation and gene expression

B

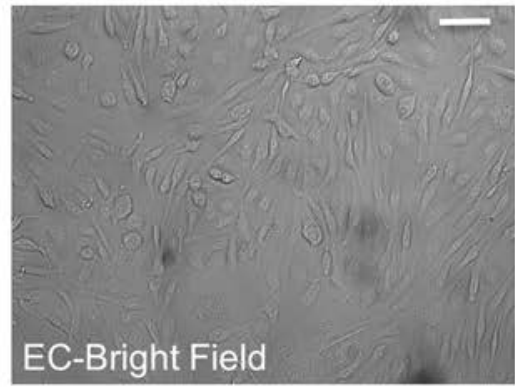

E

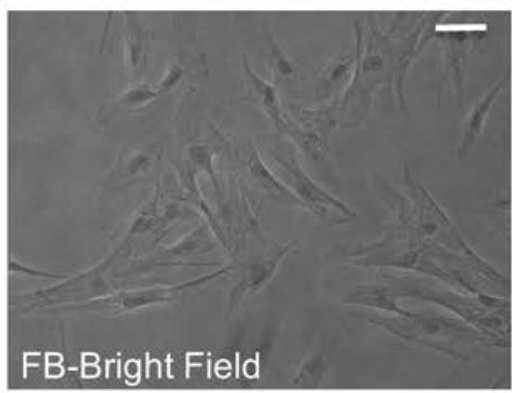

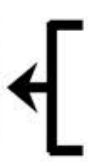

EC characterization

EC identity maintenance

Single cell gene expression

C

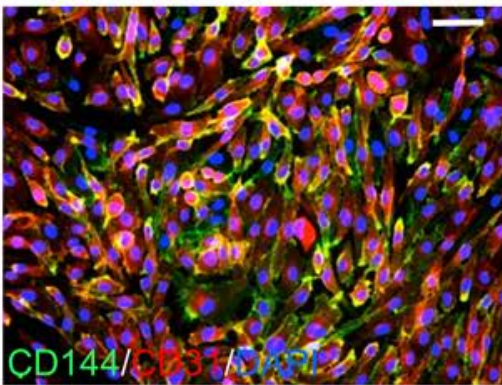

$\mathbf{F}$

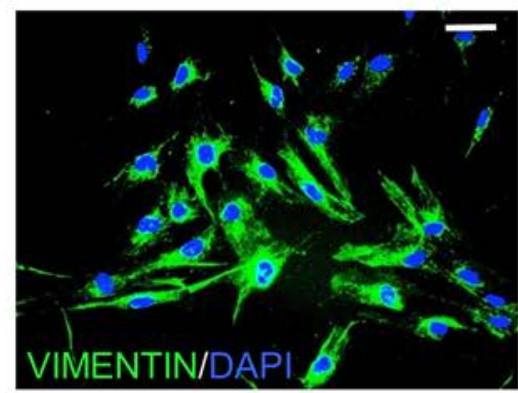

D

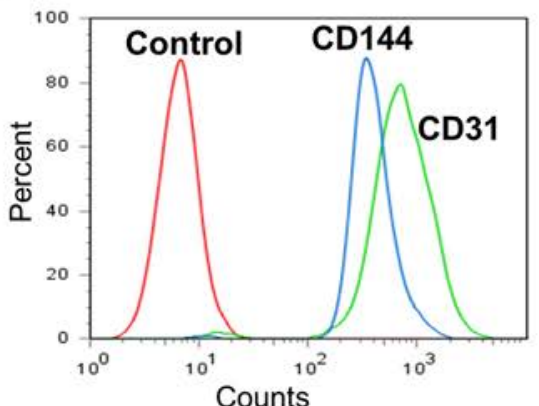

G

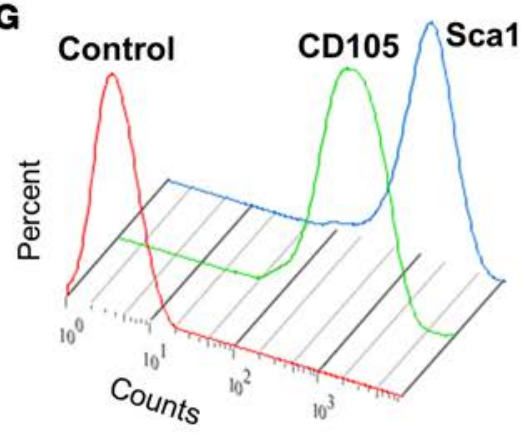

Human Sca1+ ${ }^{+} \mathrm{CPC}$

H

FV(S)

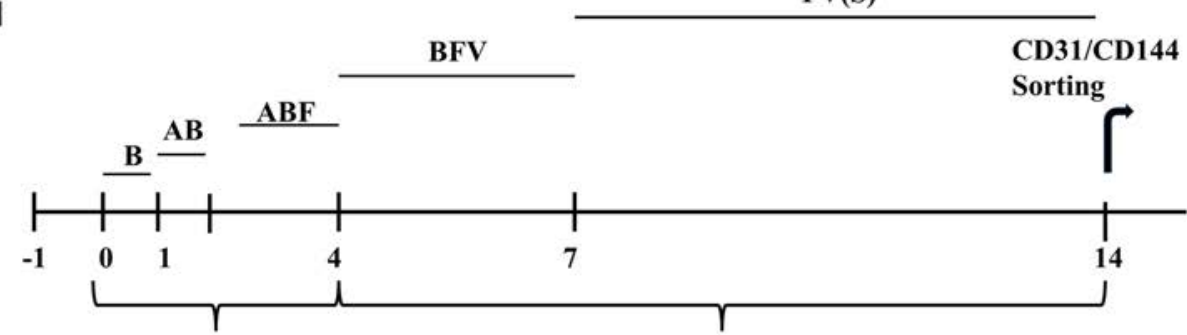

Suspension

Adherent on Matrigel

Figure 1. Isolation of fibroblasts (FBs), endothelial cells (ECs), and cardiac progenitor cells (CPCs) from the same human fetuses. (A) Schematic overview of the experimental design. Three types of somatic cells (FBs, ECs, and CPCs) were reprogrammed to iPSCs (FB-iPSCs, EC-iPSCs, and CPC-iPSCs). These iPSCs were then differentiated into endothelial cells (FB-iPSC-ECs, EC-iPSC-ECs, and CPC-iPSC-ECs). Both in vitro and in vivo assays were performed to assess the effects of donor cell source on endothelial differentiation and gene expression of human iPSCs. (B and C) Endothelial cells exhibited typical cobblestone morphology and expressed CD31 and CD144. (D) FACS analysis confirmed the high percentage of CD31+CD144+ cells in fetal ECs. (E and F) Fibroblasts were positive for mesenchymal marker vimentin. (G) Sca-1+ cardiac progenitor cells expressed CD105. (H) EC differentiation protocol by sequential administration of cytokines and growth factors. A, Activin A ( $10 \mathrm{ng} / \mathrm{ml}) ; \mathrm{B}, \mathrm{BMP} 4(20 \mathrm{ng} / \mathrm{ml}) ; \mathrm{F}, \mathrm{bFGF}(8 \mathrm{ng} / \mathrm{ml}) ; \mathrm{V}, \mathrm{VEGF}$ $(25 \mathrm{ng} / \mathrm{ml})$; and S, SB431542 (10 $\mu \mathrm{M})$. Scale bars: $50 \mu \mathrm{m}$. 
absence of basic FGF (bFGF), which consisted of the derivatives of 3 germ layers (mesoderm, ectoderm, and endoderm) in vitro (Supplemental Figure 3). When injected into immunodeficient mice, these iPSCs grew into teratomas composed of lineage descendants from all 3 germ layers (Supplemental Figure 4). Together, our results show that these iPSCs were completely reprogramed to the pluripotent state and were capable of differentiating into all somatic lineages of the body. There was no apparent difference among FB-iPSCs, CPC-iPSCs, and EC-iPSCs with regard to cellular morphology, pluripotency gene expression, and multiple lineage differentiation ability.

$E C$ differentiation in iPSCs derived from different donor cells. We next tested EC differentiation potential of EC-iPSCs, FB-iPSCs, and CPC-iPSCs in vitro. We employed an EB-based differentiation protocol as previously described (25). We differentiated ECs from very early passage iPSCs (passage $<10[\mathrm{P}<10]$ ), early passage iPSCs $(10<\mathrm{P}<20)$, and late passage iPSCs $(\mathrm{P}>20)$. In the very early passage, EC differentiation was more efficient in EC-iPSCs than in FB-iPSCs and CPC-iPSCs, as measured by the percentage of $\mathrm{CD} 31^{+}$cells in the differentiation progeny (Figure $3 \mathrm{~A}$, left panel). The biased differentiation propensity persisted in early passage iPSCs (Figure 3A, middle panel). However, when iPSCs were cultured over 20 passages, there were no significant differences in the EC differentiation efficiency among EC-iPSCs, FB-iPSCs, and CPC-iPSCs (Figure 3A, right panel). It appears that endothelial differentiation of EC-iPSCs became slightly less efficient in late-passage (Figure 3A, right panel) than early passage iPSCs (Figure 3A, left and middle panels), though EC differentiation propensity was relatively stable in FB-iPSCs and CPC-iPSCs regardless of passaging. These data suggest that somatic memory carried by iPSCs may be gradually lost during extensive passage, and late-passage iPSCs appear to share similar lineage differentiation propensity regardless of donor cell sources (18). Other factors such as telomere length erosion (26) and accumulated gene copy number variations might play a role, as well, during extensive passage of human iPSCs $(27,28)$.

The 3 types of iPSC-ECs showed typical EC identities in that they expressed membrane protein VE-cadherin (CD144) and glycoprotein von Willebrand factor (vWF) (Figure 3B, left panel) and took up LDL (Figure 3B, middle panel). In addition, these 3 types of iPSC-ECs were all capable of forming tubelike structures in the presence of extracellular matrix (Figure 3B, right panel). Because early passage EC-iPSCs displayed a higher EC differentiation efficiency, we assumed EC-iPSCs might retain EC marker gene expression in iPSCs. Thus, we next examined the gene expression of EC markers in different donor cellderived iPSCs before and after differentiation, using fetal primary ECs as a positive control for comparison. These genes included CTGF, HGF, VEGFB, KDR, NOS3, and PECAM1. Consistent with our hypothesis, EC-iPSCs quickly activated and maintained significantly higher levels of EC markers PECAM1 and NOS3 upon differentiation compared with FB-iPSCs and CPC-iPSCs (Figure 3C). In particular, undifferentiated EC-iPSCs retained slightly higher expression levels of these EC markers (Figure 3D) than FB-iPSCs and CPC-iPSCs, though these EC markers were mostly repressed in PSCs relative to primary ECs. Taken together, these results indicate that the EC-specific genes are shut down in all iPSCs but are more amenable for quick reactivation in response to differentiation signals in EC-iPSCs than CPC-iPSCs and FB-iPSCs, thus conferring a biased EC differentiation propensity.

In vitro EC identity maintenance in iPSC-ECs. The iPSC-ECs had similar characteristics as primary ECs in terms of expression of EC-specific markers (vWF, CD31, and CD144), LDL uptake, and tube formation in the presence of extracellular matrix (Figure 3B). However, the endothelial identity may not be well inherited in iPSC-ECs and ESC-ECs after prolonged culture due to their epigenetic instability and spontaneous endothelial-to-mesenchymal transition (EndoMT) (29-31). We next explored the kinetic EC identity changes of these iPSC-ECs derived from early passage iPSCs $(10<\mathrm{P}<20)$. Initially, we seeded high-purity $(>99 \%)$ CD $31^{+}$iPSC-ECs in the conventional EC culture medium, which produced a gradual loss of the $\mathrm{CD} 31^{+}$population during extensive subculture (Figure $4 \mathrm{~A}$ ). The percentage of $\mathrm{CD} 31^{+}$population dropped to less than $50 \%$ at day 20 of culture (Figure $4 \mathrm{~A}$ ). During the extensive culture, EC-iPSC-ECs consistently sustained a higher percentage of $\mathrm{CD}^{+} 1^{+}$population than FB-iPSC-ECs and CPC-iPSC-ECs. Similarly, EC-iPSC-ECs maintained a higher expression of EC-specific marker genes (PECAM1) (Figure 4B) but lower expression of FB marker genes (ACTA2 and VIM) (Figure 4, C and D). The endothelial identity of EC-iPSC-ECs was better retained, whereas EndoMT (32) was relatively ameliorated throughout the whole process of long-term culture (day 5 through day 20). Overall, EC-iPSC-ECs preserved better endothelial identity than FB-iPSC-ECs and CPC-iPSC-ECs, as evidenced by the higher expression of EC marker genes and lower expression of mesenchymal genes in vitro.

In a previous study, it was shown that TGF- $\beta$ signaling inhibition is required to maintain long-term 
A

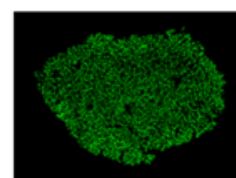

SSEA4
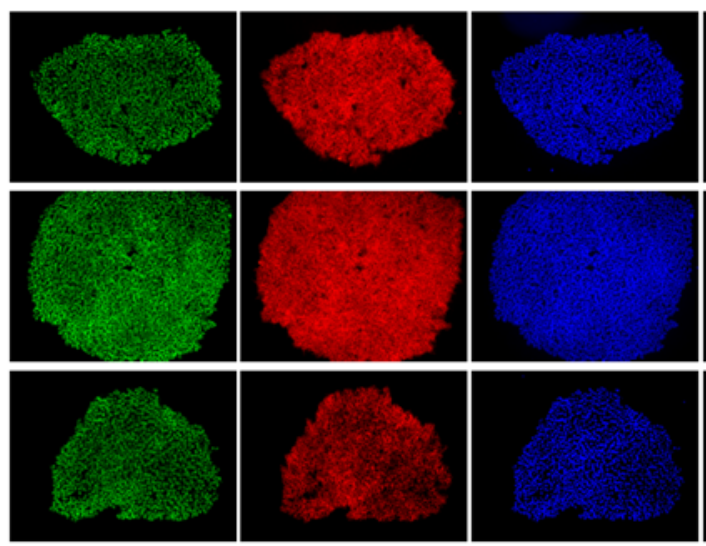

CPC-iPSCs

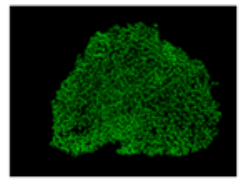

OCT4

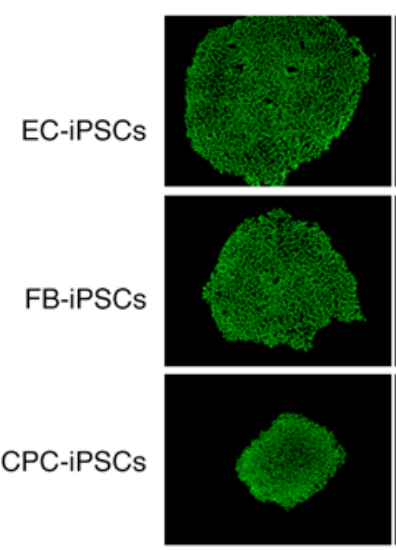

TRA-1-60
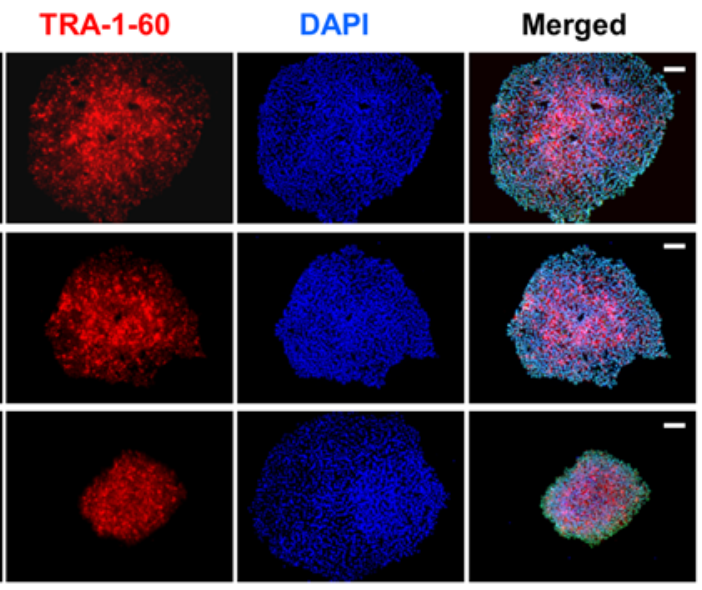

B

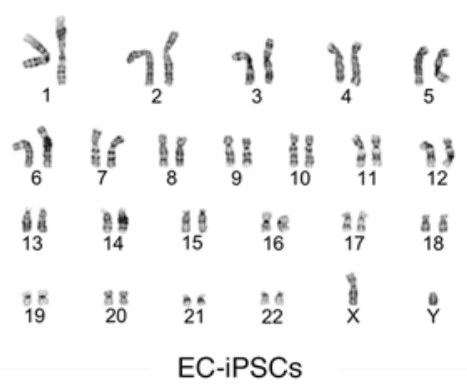

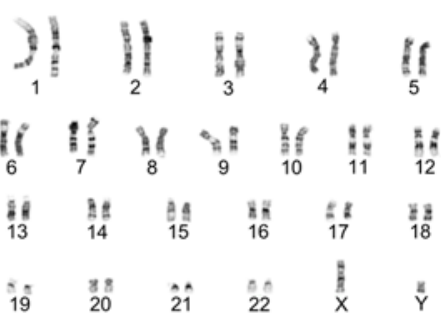

FB-iPSCs

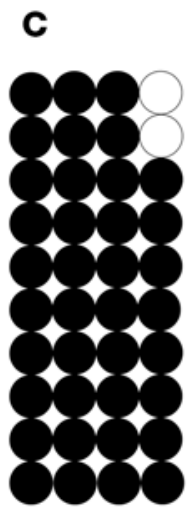

FBs

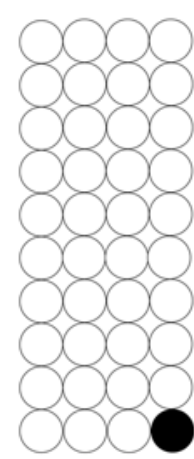

EC-iPSCs

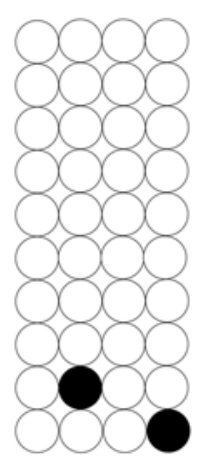

FB-iPSCs

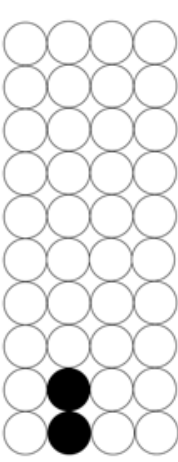

CPC-iPSCs

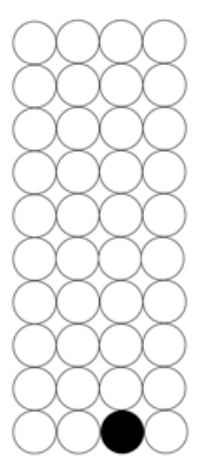

ESCs
D

$$
\begin{aligned}
& \text { 2. }
\end{aligned}
$$

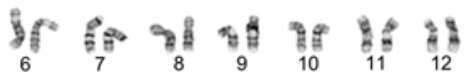

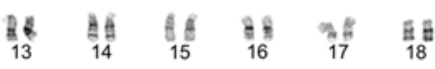

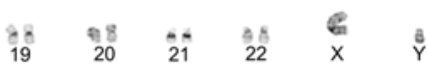

Figure 2. Human iPSC generation and characterization. (A) EC-iPSCs, FB-iPSCs, and CPC-iPSCs were positive for ESC-specific markers such as TRA-1-60, SSEA4, NANOG, and OCT4. (B) Karyotype analysis showed normal chromosomal integrity in all iPSC lines. (C) Bisulfite sequencing analysis indicated that the promoter region of NANOC was demethylated in iPSCs and ESCs but highly methylated in somatic cells. Closed circles indicate methylated cytosine, whereas open circles denote unmethylated cytosine at selected $\mathrm{Cp} G$ sites. Each column of circles represents the methylation status of designated $\mathrm{CpG}$ sites (4 CpG sites here), and each row stands for a clone containing target amplicons. (D) The quantitative PCR (qPCR) demonstrated that mRNA abundance of pluripotency gene SOX2 in these iPSCs was comparable with those in ESCs, and most mRNAs were transcribed from the endogenous pluripotent gene. Scale bars: $25 \mu \mathrm{m}$. 

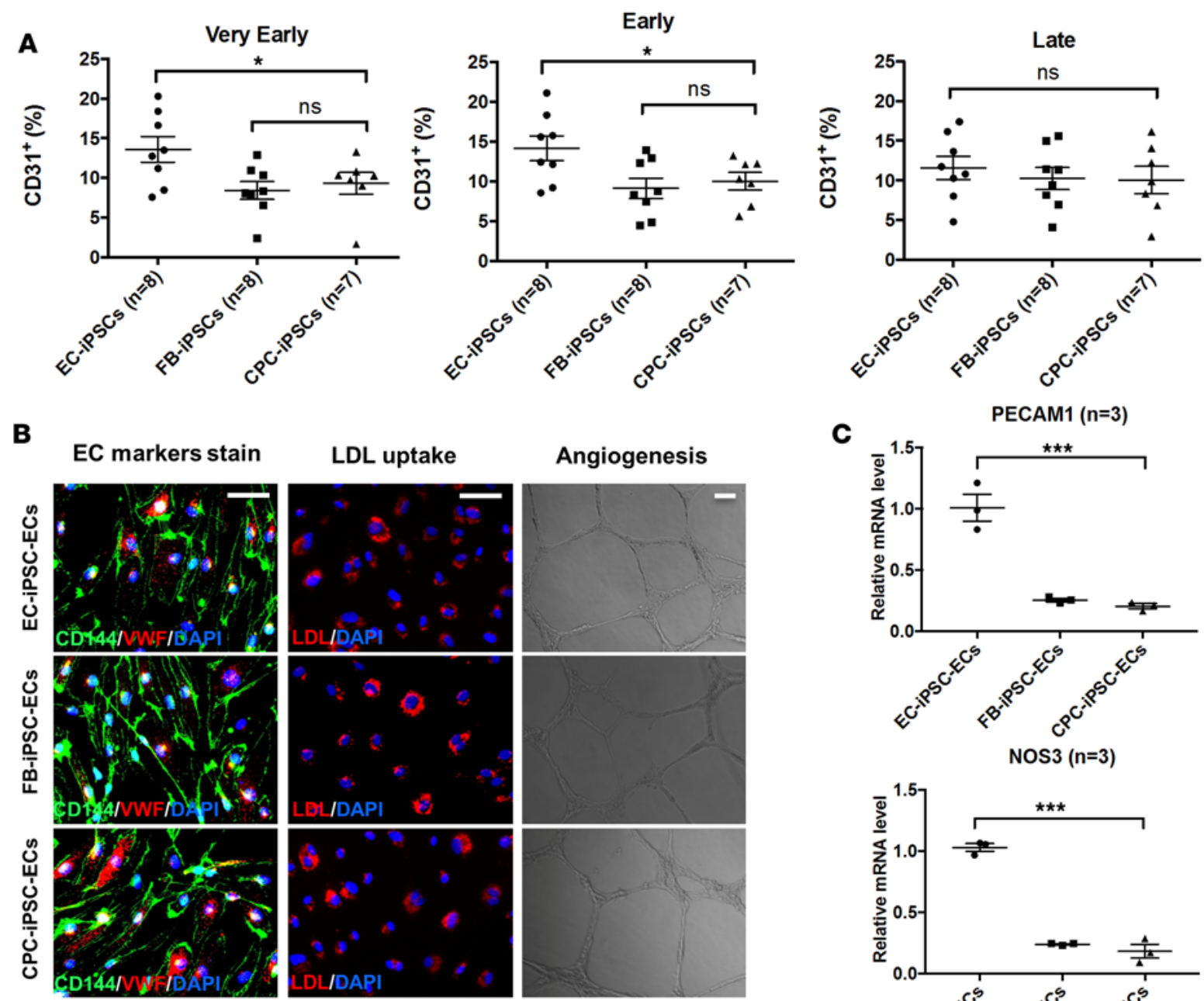

\section{Angiogenesis}
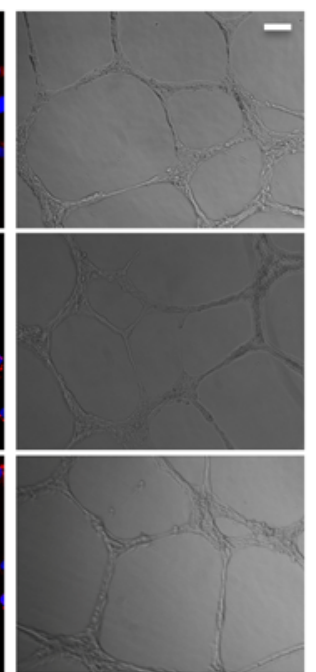

C
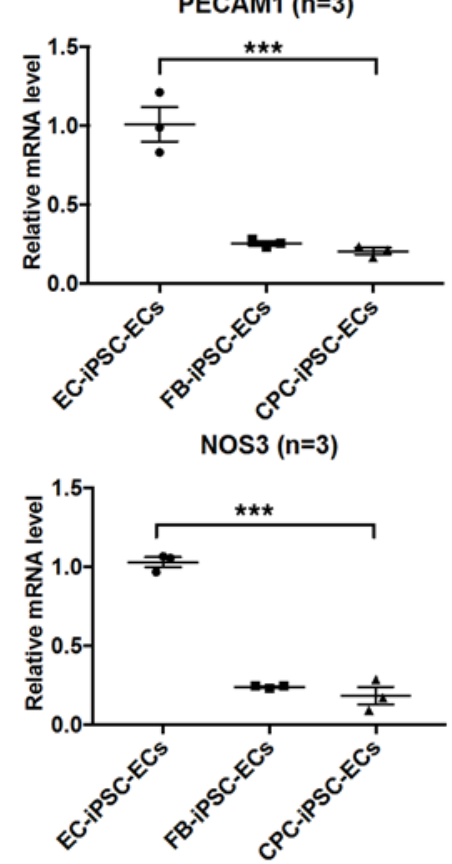
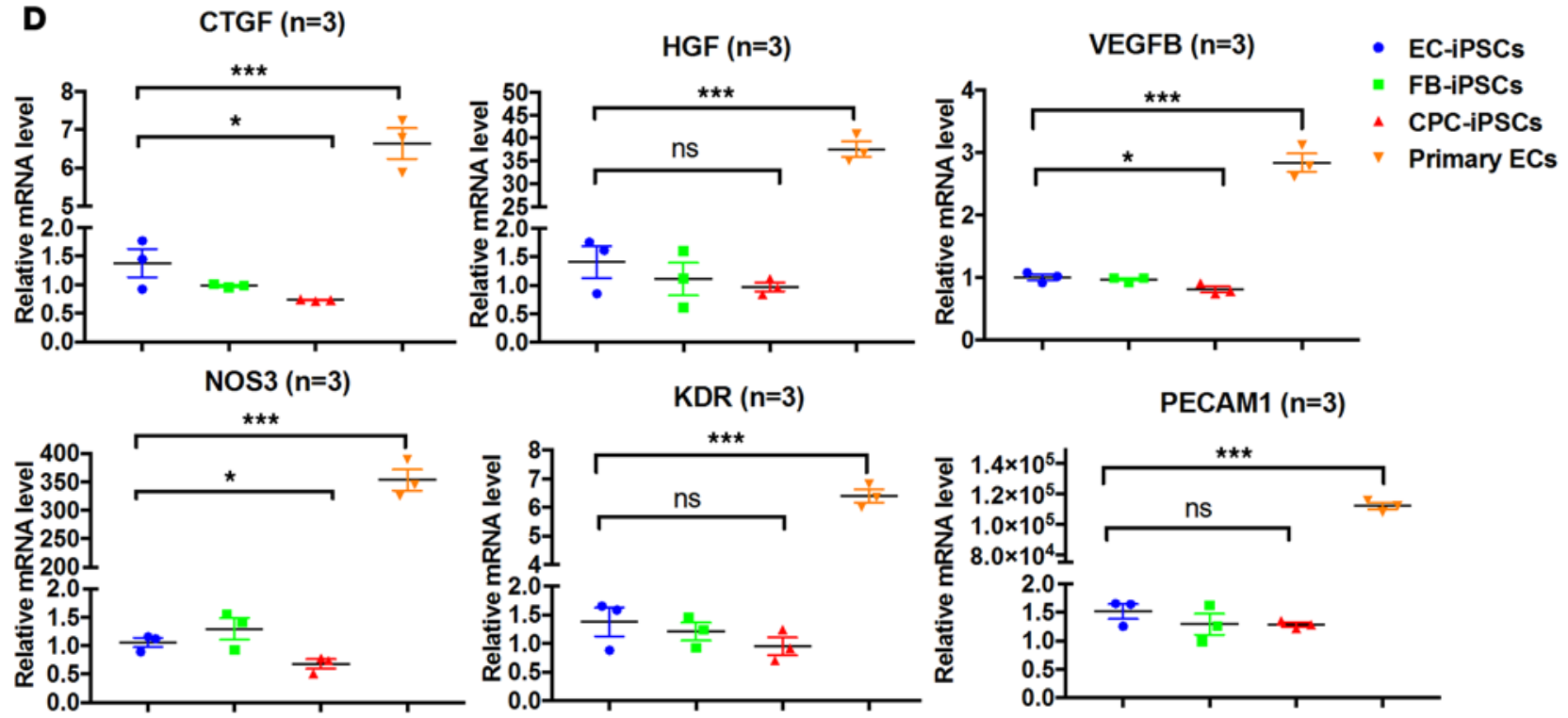
Figure 3. Characterization and comparison of endothelial differentiation in human iPSCs derived from multiple donor cell sources. (A) In very early passage $(P<10)$ and early passage $(10<P<20)$, EC-iPSCs showed a significantly higher endothelial differentiation propensity, which was identified by the percentage of $C D 31^{+}$cells. However, in late passage $(P>20)$, all 3 iPSCs displayed comparable EC differentiation efficiencies. ${ }^{*} P<0.05,1$-way ANOVA. (B) All iPSC-ECs expressed EC-specific markers CD144 and VWF (left panel). They could also uptake LDL (middle panel) and form tube-like structures on Matrigel (right panel). (C) At day 12 of induced differentiation of early passage iPSCs, PECAM1 (CD31) and NOS3 were expressed at higher levels in EC-iPSC-ECs than in FB-iPSC-ECs and CPC-iPSC-ECs. ${ }^{* *} P<0.001,1$-way ANOVA. (D) EC-specific markers were repressed in these undifferentiated iPSCs compared with primary ECs. ${ }^{*} P<0.05,{ }^{* *} P<0.001,1$-way ANOVA. Scale bars: $50 \mu \mathrm{m}$.

expansion of human ESC-ECs (29). We next investigated the response of these iPSC-ECs to a TGF- $\beta$ inhibitory molecule SB431542 using early passage iPSCs $(10<\mathrm{P}<20)$. We found that SB431542 improved the maintenance of $\mathrm{CD} 31^{+}$population in both FB-iPSC-ECs and EC-iPSC-ECs, though EC-iPSC-ECs retained a higher percentage of $\mathrm{CD} 31^{+}$population during long-term culture (Figure 4E). For lineage marker gene expression, EC-iPSC-ECs (day 20) showed a higher expression of EC-specific marker PECAM1 (Figure 4F) but a lower expression of mesenchymal markers ACTA2 (Figure 4G) and VIM (Figure 4H) than FB-iPSC-ECs after long-term culture. Similarly, in the presence of SB431542, EC-iPSC-ECs maintained a higher expression of PECAM1 (Figure 4F) and a lower expression of ACTA2 (Figure 4G) and VIM (Figure 4H) than FB-iPSC-ECs. Overall, EC-iPSC-ECs displayed better endothelial characteristic maintenance (higher percentage of $\mathrm{CD} 31^{+}$population, higher EC marker gene expression, and lower mesenchymal marker expression) than other iPSC-ECs during long-term culture, regardless of the presence of TGF- $\beta$ inhibitor SB431542.

In vivo EC identity maintenance of iPSC-ECS. To monitor iPSC derivatives after transplantation, we first made transgenic iPSCs with stable integration of a triple-fusion construct (Supplemental Figure 5A) carrying red fluorescent protein (RFP), firefly luciferase (Fluc), and herpes simplex virus thymidine kinase (HSVtk) reporter genes driven by a human ubiquitin promoter $(33,34)$. The Fluc bioluminescence signal intensity was linearly correlated $\left(\mathrm{R}^{2} \geq 0.95\right)$ with the cell number in all 3 types of iPSCs (EC-iPSCs, FB-iPSCs, and CPC-iPSCs) during long-term culture (Supplemental Figure 5B), indicating that transgenic lines could be used to quantitatively track the fate of iPSC derivatives after transplantation. We subsequently differentiated early passage iPSCs $(10<\mathrm{P}<20)$ followed by intramuscular injection of $1 \times 10^{6}$ iPSC-ECs into the ischemic hindlimb. Bioluminescence imaging confirmed engraftment of iPSC-ECs in the ischemic hindlimb at 14 days after injection. The normalized bioluminescent imaging (BLI) signals showed similar cell survival rates for different sources of iPSC-ECs (EC-iPSC-ECs, FB-iPSC-ECs, and CPC-iPSC-ECs) at day 7 and day 14 after cell transplantation (Supplemental Figure 5C).

Next, we retrieved the tagged iPSC-ECs by FACS at 2 weeks after transplantation and then characterized their endothelial identity (Figure $5 \mathrm{~A}$ ). Within the graft, a greater percentage of $\mathrm{CD} 31^{+}$population was retained by EC-iPSC-ECs compared with FB-iPSC-ECs and CPC-iPSC-ECs (Figure 5B). Single-cell qPCR confirmed that in vivo EC-iPSC-ECs had a higher expression of EC-specific markers (PECAM1, KDR, and ICAM1) and angiogenesis-related factors (IL8, TNFA, and ANGPT2) than other iPSC-ECs (Figure 5C). By contrast, mesenchymal marker ACTA2 and pluripotency marker POU5F1 had a lower expression in EC-iPSC-ECs than other iPSC-ECs, implying stronger vascular fate commitment but weaker mesenchymal fate determination in EC-iPSC-ECs. ID1 (inhibitor of DNA binding 1) is a downstream target of TGF- $\beta$ signaling and is known to modulate the differentiation and maintenance of vascular cell fate in human ESC-derived ECs $(29,35)$. We found that ID1 maintained a significantly higher mRNA abundance in EC-iPSC-ECs than in other iPSC-ECs (Figure 5D), highlighting the essential role of ID1 in rendering endothelial identity in the tissue microenvironment. Taken together, these results indicate that EC-iPSC-ECs exhibited a stronger endothelial identity when transplanted into ischemic tissue than other iPSC-ECs, accompanied by a higher expression of EC-markers and a lower rate of EndoMT.

\section{Discussion}

In the present study, we investigated the potential effect of donor cell source on human iPSC-EC differentiation and gene expression. We generated genetically identical iPSC-ECs derived from ECs, FBs, and CPCs of the same donor. The endothelial identity of these iPSC-ECs is indeed affected by the tissue-of-origin, which is reflected as tissue-specific somatic memory in terminally differentiated iPSC derivatives, especially at early passage $(10<\mathrm{P}<20)$. For example, EC-iPSC-ECs show higher endothelial marker gene expression and EC identity maintenance with extensive culture than FB-iPSC-ECs and CPC-iPSC-ECs. In addi- 
A

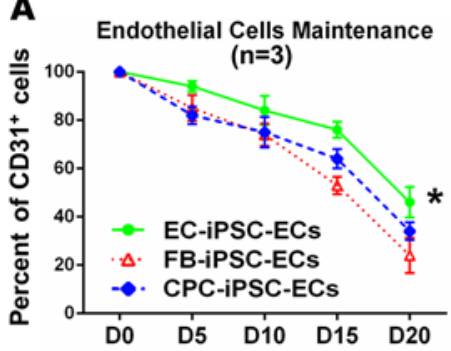

D

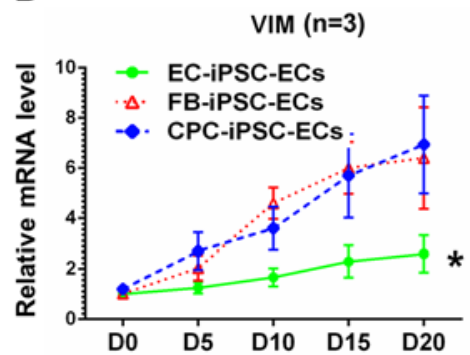

G

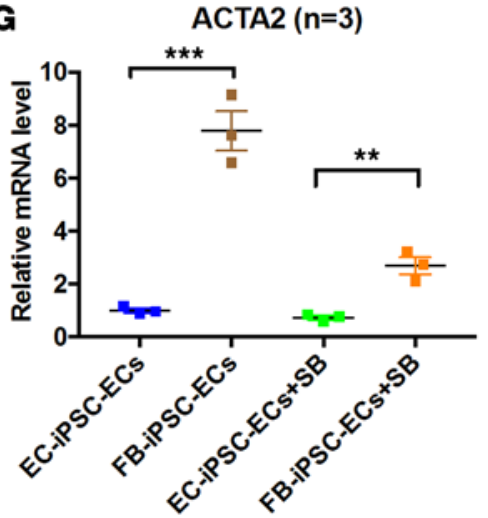

B

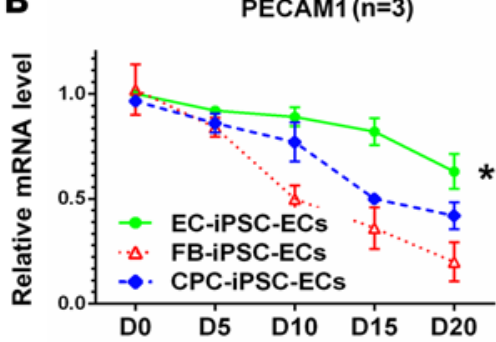

E

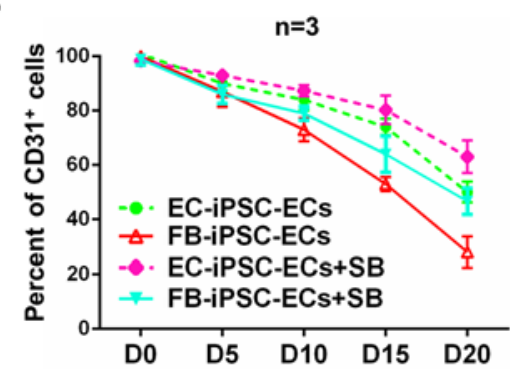

H

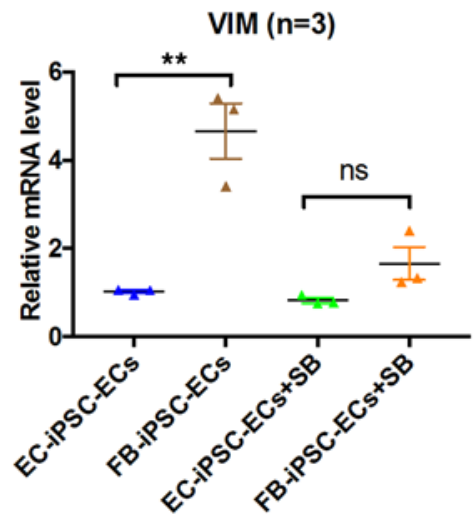

C $\operatorname{ACTA2}(n=3)$

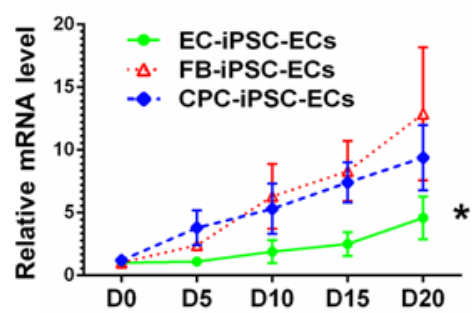

$\mathbf{F}$

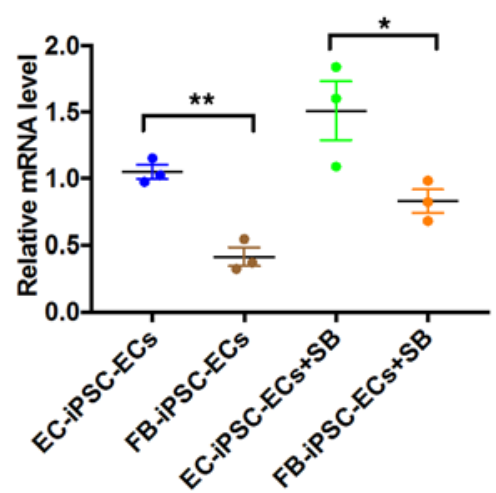

Figure 4. In vitro maintenance of endothelial identity in iPSC-ECs. (A) EC-iPSC-ECs generated from early passage iPSCs maintained a higher percentage of CD31+ cell population during long-time culture (20 days) than FB-iPSC-ECs and CPC-iPSC-ECs. ${ }^{*} P<0.05,1$-way ANOVA. (B-D) Similarly, EC-iPSC-ECs maintained higher EC marker (PECAM1) gene expression but lower expression in mesenchymal markers (ACTA2 and VIM) when compared with other iPSC-ECs during long-term culture. ${ }^{*} P<0.05$, 1 -way ANOVA. (E) TGF- $\beta$ inhibitor SB431542 boosted CD31 expression in both EC-iPSC-ECs and FB-iPSC-ECs, though EC-iPSC-ECs maintained a higher percentage of CD31+ population than FB-iPSC-ECs. (F-H) EC-iPSC-ECs (day 20) showed higher expression of EC-specific marker PECAM1 (F, left) but lower expression of mesenchymal markers ACTA2 (G, left) and VIM (H, left) compared with FB-iPSC-ECs. In the presence of SB431542, EC-iPSC-ECs maintained higher expression of PECAM1 (F, right) and lower expression of ACTA2 (G, right) and VIM (H, right) than FB-iPSC-ECs. SB, SB431542. ${ }^{*} P<0.05,{ }^{* *} P<0.01,{ }^{* *} P<0.001$, unpaired $t$-test.

tion, EC-iPSC-ECs from early passage iPSCs retain higher CD31+ population and EC-specific marker gene expression than other sources of iPSC-ECs upon transplantation into a mouse ischemic model. Our data highlight that donor cell source may affect lineage differentiation propensity and functional behaviors of human iPSC derivatives.

The epigenetic memory of the donor tissue is frequently observed in early passage human iPSCs, perhaps influencing the lineage differentiation potential and the application of iPSC derivatives in disease modeling and drug discovery $(17,36,37)$. Although previous studies reported epigenetic memory (particularly DNA methylation) in iPSCs (38), they did not test the effect of somatic memory on the characteristics of terminally differentiated cells and their therapeutic application. Here, we present data showing that donor cell source affects the lineage differentiation of iPSC-ECs both in vitro and in vivo. We found that residual somatic memory of tissue-of-origin might lead to biased differentiation efficiency in early passage human iPSCs $(10<\mathrm{P}<20)$ toward ECs in vitro. This phenomenon is also seen in iPSCs derived from other types 


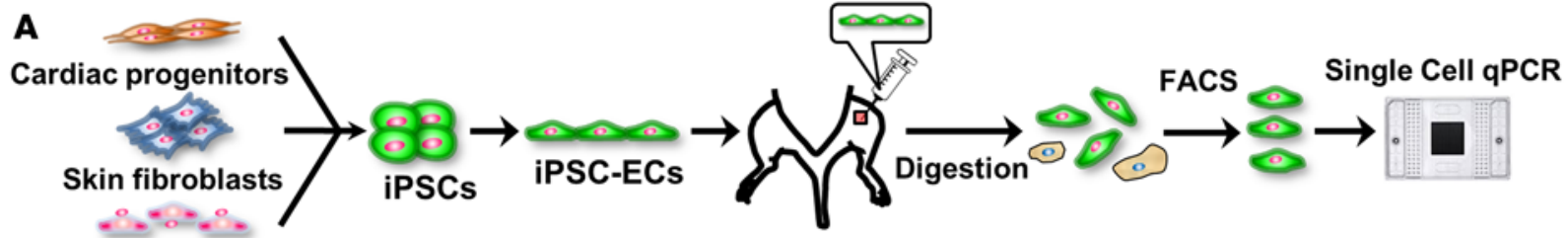

Endothelial cells

B

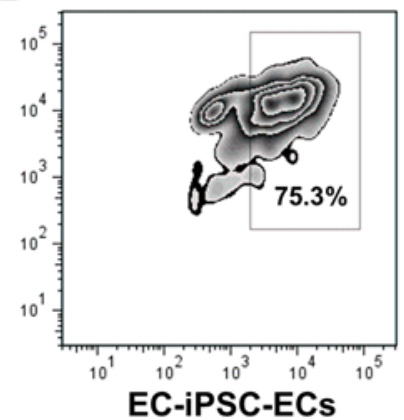

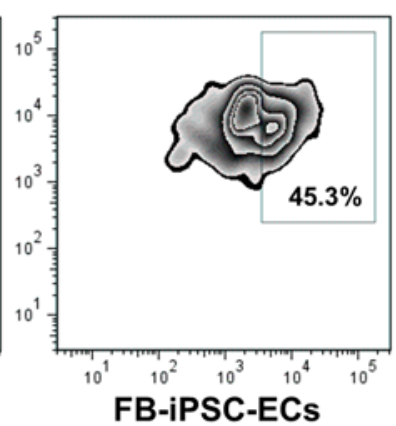

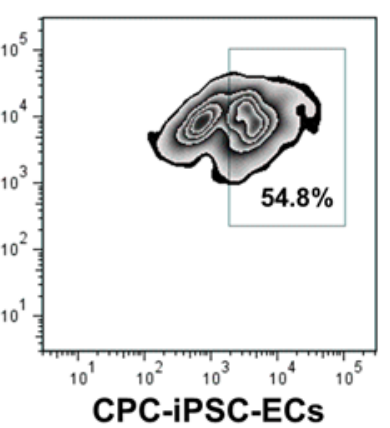

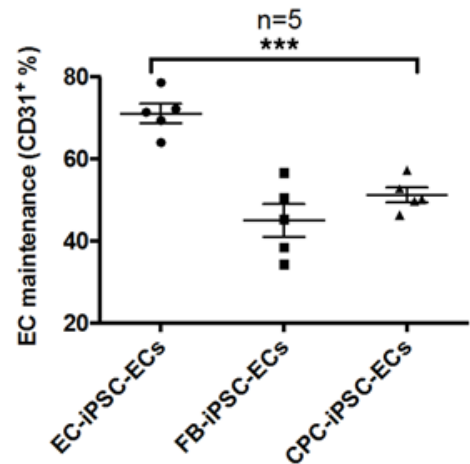

C
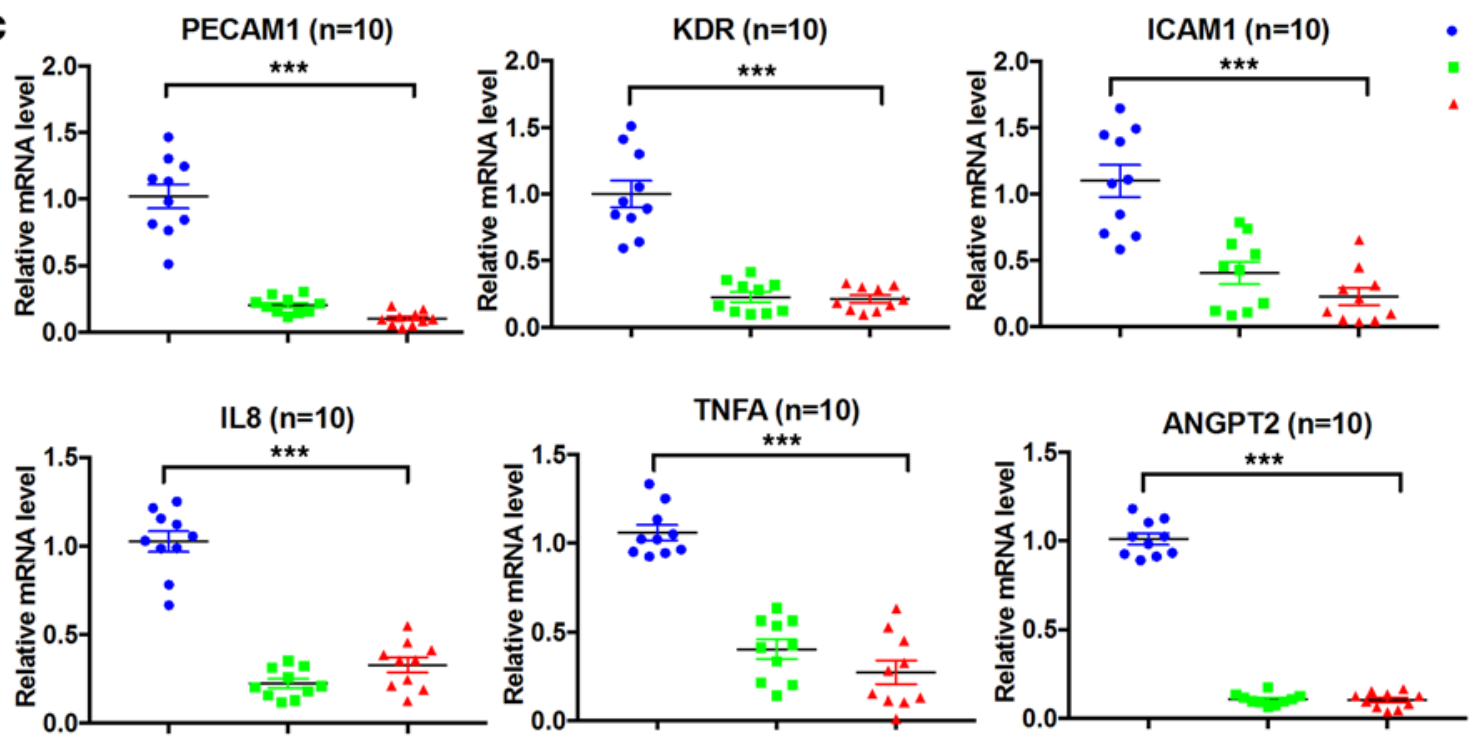

D
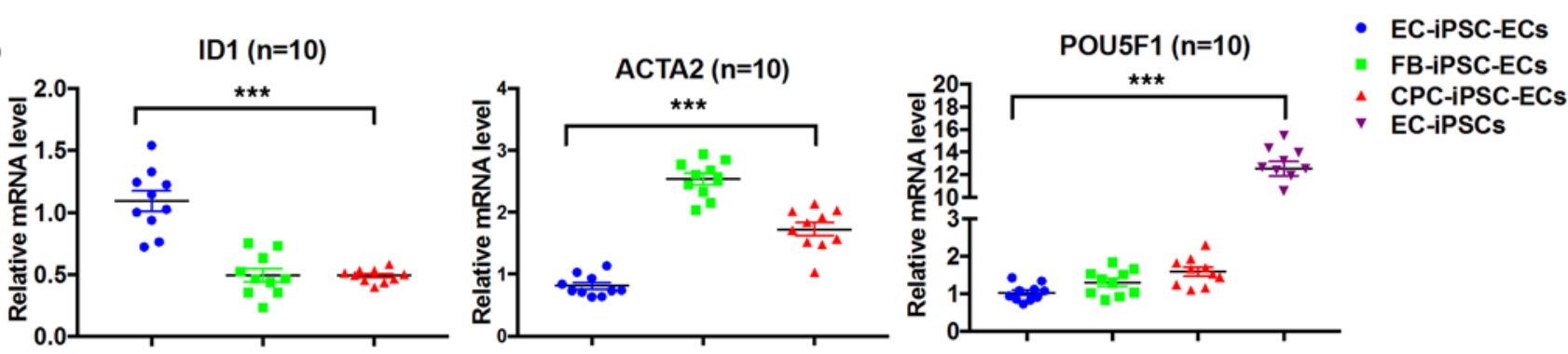

Figure 5. In vivo maintenance of endothelial identity in iPSC-ECs. (A) Schematic procedure for transplantation using different sources of iPSC-ECs generated from early passage $(10<\mathrm{P}<20)$ iPSCs using murine hindlimb ischemia model. The injected iPSC-ECs were retrieved by enzymatic digestion and sorted by FACS in 2 weeks. (B) The EC-iPSC-ECs showed higher percentage of CD31+/CD144+ population than FB-iPSC-ECs and CPC-iPSC-ECs residing in vivo. The $x$ and $y$ axes represent cell count. (C) Single-cell qPCR analysis indicated that the recovered EC-iPSC-ECs expressed higher levels of EC-specific markers (PECAM1 and KDR) and angiogenesis-associated factors (ICAM1, IL8, TNFA, and ANGPT2) than FB-iPSC-ECs and CPC-iPSC-ECs. (D) By contrast, EC-iPSC-ECs had significantly less mRNA abundance of mesenchymal marker ACTA2 and pluripotency marker POU5F1 but increased expression of inhibitor of DNA-binding 1 (ID1), which is required for long-term expansion and maintenance of stem cell-derived ECs. ${ }^{* * *} P<0.001,1$-way ANOVA. 
of somatic cells, such as hematopoietic, myogenic cells, CPCs, and insulin-producing $\beta$ cells $(15,18,36$, 39). The original lineage-determining genes and tissue-specific genes are more primed to commit toward differentiation and phenotypic maturation in the same tissue-of-origin iPSCs than other iPSCs. By contrast, this biased differentiation is absent in nuclear transfer-derived human PSCs $(15,16)$, the epigenetic memory of which is more completely erased during reprogramming. Therefore, for reprogramming based on transcription factors, early passage human iPSCs retain the somatic memory of their tissue-of-origin, which can substantially influence the differentiation potential and subsequent cell fate commitment.

Patient-specific iPSC-ECs are a promising cell source for disease modeling, drug screening, and vascular tissue engineering (4, 40-42). Human iPSC-ECs can promote in vivo angiogenesis when implanted into SCID mice (30), as shown by improved blood perfusion in a hindlimb ischemia model (43). In addition, human iPSC-ECs closely resemble native ECs in global gene expression and metabolic profiles (44). Although limited gene expression variation has been observed among multiple lines of iPSC-ECs (45), we did notice a higher percentage of $\mathrm{CD} 31^{+}$cells and endothelial marker gene expression in recovered EC-iPSC-ECs (derived from early passage iPSCs) compared with the other sources of iPSC-ECs after transplantation to mouse hindlimbs. As these iPSCs are genetically identical, we hypothesize that the therapeutic discrepancy may be caused by the tissue-of-origin epigenetic memory.

EndoMT is a process of cellular transdifferentiation in which ECs lose their vascular identity and gradually acquire the phenotype of mesenchymal cells, such as by expressing mesenchymal markers like smooth muscle actin and vimentin (32). The EndoMT takes place during normal embryonic development of the heart and contributes to cardiac fibrosis in chronic heart disease (46). The EndoMT is initiated by TGF- $\beta$ signaling, and inhibition of TGF- $\beta$ is required for the maintenance and expansion of human and mouse ESC-ECs and iPSC-ECs in vitro $(29,47)$. In this study, we observed a similar EndoMT incidence in long-term culture of human iPSC-ECs. Under these conditions, EC-iPSC-ECs are more resistant to EndoMT and exhibit higher endothelial marker expression and lower mesenchymal marker expression than other sources of iPSC-ECs. Similarly, TGF- $\beta$ inhibitor improves the endothelial identity conservation of EC-iPSC-ECs compared with FB-iPSC-ECs during long-term culture. It is possible that EC-iPSCECs may be epigenetically more similar to primary ECs that are naturally invulnerable to EndoMT in the absence of TGF- $\beta$ inhibitor. Hence, the endothelial-specific somatic memory transmitted to EC-iPSC-ECs may confer their innate resistance to EndoMT.

In conclusion, by generating genetically matched iPSC derivatives, we demonstrate that cellular origin of early passage human iPSCs affects their in vitro and in vivo endothelial identity. Generating iPSC-ECs from primary ECs may be a promising way to improve human iPSC-based regenerative therapy in the future.

\section{Methods}

Further information can be found in Supplemental Methods.

Generation of human iPSCs. Human FBs, ECs (48), and CPCs (21) were isolated from 2 fetal donors and passaged 3-5 times before lentiviral infection with Yamanaka factors OCT4, SOX2, KLF4, and C-MYC. Colonies with typical stem cell morphology were lifted and replated on Matrigel-coated plates (BD Biosciences) for maintenance in mTeSR-1 medium (STEMCELL Technologies) as previously described (25).

EC differentiation. For endothelial differentiation (Figure $1 \mathrm{H}$ ), EBs were formed in an ESC medium depleted of recombinant human FGF-2 (rhFGF-2) (R\&D Systems) on day 0. On day 1, the medium was supplemented with $20 \mathrm{ng} / \mathrm{ml}$ Activin A (R\&D Systems) and $20 \mathrm{ng} / \mathrm{ml} \mathrm{BMP4}$ (R\&D Systems). On day 2, the medium was supplemented with $10 \mathrm{ng} / \mathrm{ml} \mathrm{rhFGF}-2,20 \mathrm{ng} / \mathrm{ml}$ Activin A, and $20 \mathrm{ng} / \mathrm{ml} \mathrm{BMP} 4$. On day 4, the EBs were seeded onto Matrigel-coated dishes, and the medium was supplemented with $10 \mathrm{ng} /$ $\mathrm{ml}$ rhFGF-2 and $20 \mathrm{ng} / \mathrm{ml}$ VEGF (R\&D Systems) for EC expansion. On day 14, the EBs were harvested by Accutase (Sigma-Aldrich) and sorted using anti-CD31 monoclonal antibody (BD Biosciences, catalog 558068). The differentiation efficiency was calculated by the number of $\mathrm{CD} 31^{+} \mathrm{CD} 144^{+}$cells divided by the number of total differentiated cells, excluding dead cells at the end of induced differentiation.

Hindlimb ischemia model. A murine hindlimb ischemia model was employed to evaluate the therapeutic potential of iPSC-ECs. Briefly, unilateral hindlimb ischemia was induced by the ligation of the femoral artery of 1 hindlimb of 10-month-old female SCID beige mice. Mice were randomly divided into 4 groups: (i) FB-iPSC-ECs, (ii) EC-iPSC-ECs, (iii) CPC-iPSC-ECs, and (iv) saline. One million iPSC-ECs (derived from iPSCs at passage 15-20) were i.m. injected around the ischemic area. After transplantation, cell survival rates were monitored by BLI of Fluc reporter gene as previously described (49). The injected 
hindlimbs were enzymatically digested, and single cells were retrieved using FACS sorting in 2 weeks. The single cells were subjected to single-cell qPCR assay.

Microfluidic single-cell gene expression profiling. Single cells were sorted into wells containing $10 \mu \mathrm{l}$ of reaction buffer provided in a CellsDirect One-Step qRT-PCR kit (Invitrogen). Reverse transcription and specific transcript preamplification were performed using SuperScript III Reverse Transcriptase and Platinum Taq Mix (ThermoFisher Scientific). The program was run in an Applied Biosystems Veriti thermal cycler as follows: $50^{\circ} \mathrm{C}$ for 15 minutes, $70^{\circ} \mathrm{C}$ for 2 minutes, and $94^{\circ} \mathrm{C}$ for 2 minutes; $94^{\circ} \mathrm{C}$ for 15 seconds, $60^{\circ} \mathrm{C}$ for 30 seconds, and $68^{\circ} \mathrm{C}$ for 45 seconds for 18 cycles; followed by $68^{\circ} \mathrm{C}$ for 7 minutes. The preamplified cDNA was loaded onto Biomark 48.48 Dynamic Array chips (Fluidigm) using the Nanoflex IFC controller (Fluidigm). qPCR was performed on a BioMark HD System (Fluidigm), and data were processed by BioMark Real-Time qPCR Analysis software.

Statistics. All experiments were performed at least 3 times, and data were analyzed using 1-way ANOVA for multiple comparisons. Pairwise comparisons were performed using a 2-tailed Student's $t$ test. $P$ values less than 0.05 are considered statistically significant. Numeric data (FACS and qPCR) were presented as mean $\pm \mathrm{SEM}$ in the figures.

Study approval. All protocols in this study were approved by the Stanford University Human Subjects Research IRB and Administrative Panel on Laboratory Animal Care (APLAC).

\section{Author contributions}

SH, MTZ, and JCW conceived and performed the experiments and wrote the manuscript. FJ and NYS performed the molecular experiments and analyzed the data. WHL helped with single-cell qPCR and data analysis. HC assisted in figure preparations. MPS and JCW supervised this study and provided advice and funding support.

\section{Acknowledgments}

We are grateful to Stephen Quake for providing technical and instrumental support for single-cell qPCR. We thank Caressa Chen and Larry Bowen for critical editing of the manuscript. This study was supported by an AHA Postdoctoral Fellowship 10POST3730079 (to S. Hu); an AHA Established Investigator Award 14420025 (to J.C. Wu); and NIH grants R24 HL117756, R01 HL113006, R01 HL130020 (to J.C. Wu) and P01 GM099130 (to M.P. Snyder and J.C. Wu). M.T. Zhao is partially supported by a Stanford Cardiovascular Institute Postdoctoral Fellowship and Research Award from the Lucile Packard Foundation for Children's Health, Stanford NIH-NCATS-CTSA UL1 TR001085, and Child Health Research Institute of Stanford University.

Address correspondence to: Joseph C. Wu, Lorry I. Lokey Stem Cell Research Building, 265 Campus Drive, Room G1120B, Stanford, California 94305-5454, USA. Phone: 650.736.2246; E-mail: joewu@ stanford.edu. Or to: Shijun Hu, 708 Renmin Road, Building 1, Room 1609, Suzhou, Jiangsu 215007, China. Phone: 86.512.67781897; E-mail: shijunhu@suda.edu.cn

\footnotetext{
1. Mozaffarian D, et al. Heart disease and stroke statistics--2015 update: a report from the American Heart Association. Circulation. 2015;131(4):e29-322.

2. Hansson GK. Inflammation, atherosclerosis, and coronary artery disease. N Engl J Med. 2005;352(16):1685-1695.

3. Jaffe EA, Nachman RL, Becker CG, Minick CR. Culture of human endothelial cells derived from umbilical veins. J Clin Invest. 1973;52(11):2745-2756.

4. Wilson HK, Canfield SG, Shusta EV, Palecek SP. Concise review: tissue-specific microvascular endothelial cells derived from human pluripotent stem cells. Stem Cells. 2014;32(12):3037-3045.

5. Clayton ZE, Sadeghipour S, Patel S. Generating induced pluripotent stem cell derived endothelial cells and induced endothelial cells for cardiovascular disease modelling and therapeutic angiogenesis. Int J Cardiol. 2015;197:116-122.

6. Grskovic M, Javaherian A, Strulovici B, Daley GQ. Induced pluripotent stem cells — opportunities for disease modelling and drug discovery. Nat Rev Drug Discov. 2011;10(12):915-929.

7. Tada M, Takahama Y, Abe K, Nakatsuji N, Tada T. Nuclear reprogramming of somatic cells by in vitro hybridization with ES cells. Curr Biol. 2001;11(19):1553-1558.

8. Cowan CA, Atienza J, Melton DA, Eggan K. Nuclear reprogramming of somatic cells after fusion with human embryonic stem cells. Science. 2005;309(5739):1369-1373.

9. Wilmut I, Schnieke AE, McWhir J, Kind AJ, Campbell KH. Viable offspring derived from fetal and adult mammalian cells Nature. 1997;385(6619):810-813.

10. Tachibana M, et al. Human embryonic stem cells derived by somatic cell nuclear transfer. Cell. 2013;153(6):1228-1238.
} 
11. Takahashi K, Yamanaka S. Induction of pluripotent stem cells from mouse embryonic and adult fibroblast cultures by defined factors. Cell. 2006;126(4):663-676.

12. Yu J, et al. Induced pluripotent stem cell lines derived from human somatic cells. Science. 2007;318(5858):1917-1920.

13. Chin $\mathrm{MH}$, et al. Induced pluripotent stem cells and embryonic stem cells are distinguished by gene expression signatures. Cell Stem Cell. 2009;5(1):111-123.

14. Guenther MG, et al. Chromatin structure and gene expression programs of human embryonic and induced pluripotent stem cells. Cell Stem Cell. 2010;7(2):249-257.

15. Kim K, et al. Epigenetic memory in induced pluripotent stem cells. Nature. 2010;467(7313):285-290.

16. $\mathrm{Ma} \mathrm{H}$, et al. Abnormalities in human pluripotent cells due to reprogramming mechanisms. Nature. 2014;511(7508):177-183

17. Kim K, et al. Donor cell type can influence the epigenome and differentiation potential of human induced pluripotent stem cells. Nat Biotechnol. 2011;29(12):1117-1119.

18. Polo JM, et al. Cell type of origin influences the molecular and functional properties of mouse induced pluripotent stem cells. Nat Biotechnol. 2010;28(8):848-855

19. Rouhani F, Kumasaka N, de Brito MC, Bradley A, Vallier L, Gaffney D. Genetic background drives transcriptional variation in human induced pluripotent stem cells. PLoS Genet. 2014;10(6):e1004432.

20. Choi J, et al. A comparison of genetically matched cell lines reveals the equivalence of human iPSCs and ESCs. Nat Biotechnol. 2015;33(11):1173-1181.

21. Smits AM, et al. Human cardiomyocyte progenitor cells differentiate into functional mature cardiomyocytes: an in vitro model for studying human cardiac physiology and pathophysiology. Nat Protoc. 2009;4(2):232-243.

22. Handgretinger R, Kuci S. CD133-positive hematopoietic stem cells: from biology to medicine. Adv Exp Med Biol. 2013;777:99111.

23. Urbich C, Dimmeler S. Endothelial progenitor cells: characterization and role in vascular biology. Circ Res. 2004;95(4):343-353.

24. Asahara T, et al. Isolation of putative progenitor endothelial cells for angiogenesis. Science. 1997;275(5302):964-967.

25. Hu S, et al. MicroRNA-302 increases reprogramming efficiency via repression of NR2F2. Stem Cells. 2013;31(2):259-268.

26. Marion RM, et al. Telomeres acquire embryonic stem cell characteristics in induced pluripotent stem cells. Cell Stem Cell. 2009;4(2):141-154.

27. Hussein SM, et al. Copy number variation and selection during reprogramming to pluripotency. Nature. 2011;471(7336):58-62.

28. Laurent LC, et al. Dynamic changes in the copy number of pluripotency and cell proliferation genes in human ESCs and iPSCs during reprogramming and time in culture. Cell Stem Cell. 2011;8(1):106-118.

29. James D, et al. Expansion and maintenance of human embryonic stem cell-derived endothelial cells by TGF $\beta$ inhibition is Id1 dependent. Nat Biotechnol. 2010;28(2):161-166.

30. Li Z, Hu S, Ghosh Z, Han Z, Wu JC. Functional characterization and expression profiling of human induced pluripotent stem cell- and embryonic stem cell-derived endothelial cells. Stem Cells Dev. 2011;20(10):1701-1710.

31. Kokudo T, Suzuki Y, Yoshimatsu Y, Yamazaki T, Watabe T, Miyazono K. Snail is required for TGFß-induced endothelial-mesenchymal transition of embryonic stem cell-derived endothelial cells. J Cell Sci. 2008;121(pt 20):3317-3324.

32. Piera-Velazquez S, Li Z, Jimenez SA. Role of endothelial-mesenchymal transition (EndoMT) in the pathogenesis of fibrotic disorders. Am J Pathol. 2011;179(3):1074-1080.

33. Cao F, et al. In vivo visualization of embryonic stem cell survival, proliferation, and migration after cardiac delivery. Circulation. 2006;113(7):1005-1014.

34. Wang Y, et al. Genome editing of human embryonic stem cells and induced pluripotent stem cells with zinc finger nucleases for cellular imaging. Circ Res. 2012;111(12):1494-1503.

35. Ruzinova MB, Benezra R. Id proteins in development, cell cycle and cancer. Trends Cell Biol. 2003;13(8):410-418.

36. Bar-Nur O, Russ HA, Efrat S, Benvenisty N. Epigenetic memory and preferential lineage-specific differentiation in induced pluripotent stem cells derived from human pancreatic islet $\beta$ cells. Cell Stem Cell. 2011;9(1):17-23.

37. Lister R, et al. Hotspots of aberrant epigenomic reprogramming in human induced pluripotent stem cells. Nature. 2011;471(7336):68-73

38. Ohi Y, et al. Incomplete DNA methylation underlies a transcriptional memory of somatic cells in human iPS cells. Nat Cell Biol. 2011;13(5):541-549.

39. Sanchez-Freire V, et al. Effect of human donor cell source on differentiation and function of cardiac induced pluripotent stem cells. J Am Coll Cardiol. 2014;64(5):436-448.

40. Samuel R, et al. Generation of functionally competent and durable engineered blood vessels from human induced pluripotent stem cells. Proc Natl Acad Sci U S A. 2013;110(31):12774-12779.

41. Theodoris CV, et al. Human disease modeling reveals integrated transcriptional and epigenetic mechanisms of NOTCH1 haploinsufficiency. Cell. 2015;160(6):1072-1086

42. Wilson KD, Wu JC. Induced pluripotent stem cells. JAMA. 2015;313(16):1613-1614.

43. Rufaihah AJ, et al. Endothelial cells derived from human iPSCS increase capillary density and improve perfusion in a mouse model of peripheral arterial disease. Arterioscler Thromb Vasc Biol. 2011;31(11):e72-e79.

44. Patsch C, et al. Generation of vascular endothelial and smooth muscle cells from human pluripotent stem cells. Nat Cell Biol. 2015;17(8):994-1003.

45. White MP, et al. Limited gene expression variation in human embryonic stem cell and induced pluripotent stem cell-derived endothelial cells. Stem Cells. 2013;31(1):92-103.

46. Zeisberg EM, et al. Endothelial-to-mesenchymal transition contributes to cardiac fibrosis. Nat Med. 2007;13(8):952-961.

47. Israely E, et al. Akt suppression of TGF $\beta$ signaling contributes to the maintenance of vascular identity in embryonic stem cell-derived endothelial cells. Stem Cells. 2014;32(1):177-190.

48. Donnini D, Perrella G, Stel G, Ambesi-Impiombato FS, Curcio F. A new model of human aortic endothelial cells in vitro. Biochimie. 2000;82(12):1107-1114.

49. Hu S, et al. Novel microRNA prosurvival cocktail for improving engraftment and function of cardiac progenitor cell transplantation. Circulation. 2011;124(11 suppl):S27-S34. 